\title{
不確実な観測機構を有する線形連続時間系の状態推定
}

\author{
藤重悟・片山徹・椹木義一 \\ 京都大学工学部 京都市左京区吉田本町 \\ (昭和 48 年 2 月 28 日 .受付)
}

\section{State Estimation for Linear Continuous-Time Systems with Interrupted Observations}

\author{
Satoru Fujishige, Tohru Katayama and Yoshikazu Sawaragi \\ (Faculty of Engineering, Kyoto University, Kyoto) \\ (Received February 28, 1973)
}

\begin{abstract}
A minimum variance estimator is derived for linear continuous-time systems with an interrupted observation mechanism which is characterized in terms of the jump Markov process taking on the values of 0 or 1 . The approach adopted is that we express the jump Markov process in terms of the initial value and jump times instead of the instantaneous values and then apply Lainiotis' formula regarding the initial value and jump times as unknown system parameters. The resultant optimal algorithm is infinite dimensional, so that feasible approximate estimator algorithms are presented for practical implementation.
\end{abstract}

\section{1. まえがき}

最近, Kalman-Bucy フィルタ1,2)の拡張として, 不 確実な観測機構を有する線形系の状態推定問題 3 9) お よび，推定と検定の結合9,10)などに関して多くの研究 がなされてきている。しかし，こ和らの研究はほとえ ぞ離散時間系に関するもので，連続時間系については システムに未知定数パラメータを含場合のみを考察

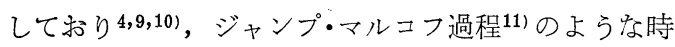
変未知パラメータ它含导場合の推定問題は, まだ研究 されていない。

著者らは，すでに不確実な観測機構を有する線形離 散時間系の状態推定問題について報告した8) が，本論 交では，連続時間系に対する同様の間題を考察する。 システムの状態は, 通常の拡散過程によって記述され ているが，不確実な観測は 0 または 1 の值をとるジャ ンプ・マルコフ過程によって特徴づけられているので, 観測值はジャンプ・マルコフ過程の成分を含えでいる. このため, Jaffer-Gupta による離散時間系の最適推定 アルゴリズム ${ }^{6)}$ において，サンプル間隔を 0 亿近づけ ても, 連続時間系に対する最適推定アルゴリズムを得 ることは期待できない，本論交では，ジャンプ・マル
コフ過程をその初期值とジャンプ時刻によって表現 し，それらをシステムの未知パラメータと考光，まず Lainiotisの公式91学適用し，ついでその結果にある種 の極限操作を施すことによって, 最適推定アルゴリズ ムを得ている。

本論交では，2節において，不確実な観測機構定有す る線形連続時間系の状態推定問題を正確に定式化し， 3 節において最小分散推定アルゴリズムが導出され る. 得られた最適推定アルゴリズムは無限次元となっ ているので, 4 節において, 実行可能な近似最適推定了 ルゴリズムが提案される。

\section{2. 問 題の記 述}

確率空間 $(\Omega, \beta, P)$ が与光ら就ていると仮定する。 ただし， $\Omega$ 浪根元事象 $\omega$ からなるある集合， $\beta$ は $\Omega$ の 部分集合からなる $\sigma$-集合体，そして，Pは $\beta$ の上飞 定義された確率測度である。.以下では，すべての確率 変数梳，乙の確率空間上に定義されているとする．こ こで対象とするシステムは, Ito 型確率微分方程式

$$
d x(t, \omega)=F(t) x(t, \omega) d t+d w(t, \omega), t \geq 0
$$

で与えられ，観則は

$$
d y(t, \omega)=\gamma(t, \omega) H(t) x(t, \omega) d t+
$$




$$
+d v(t, \omega), t \geq 0
$$

で行なわれるとする。ここに， $x(t, \omega)$ は $n \times 1$ 状態べ クトル, $y(t, \omega)$ は $p \times 1$ 観測ベクトル, $F(t)$ は $n \times n$ 状態遷移行列, $H(t)$ は $p \times n$ 観測行列, そして, $w(t$, $\omega), v(t, \omega)$ は，それぞれ

$$
\begin{aligned}
& w(0, \omega)=0, \quad E\{w(t, \omega)\}=0, \\
& E\left\{w(t, \omega) w^{\prime}(s, \omega)\right\}=\int_{0}^{\min (t, s)} Q(u) d u, \\
& v(0, \omega)=0, \quad E\{v(t, \omega)\}=0, \\
& E\left\{v(t, \omega) v^{\prime}(s, \omega)\right\}=\int_{0}^{\min (t, s)} R(u) d u
\end{aligned}
$$

を満足する $n$ 次元および $p$ 次元 Wiener 過程である. 初期状態 $x(0, \omega)$ 注, 平均值, $\hat{x}(0 \mid 0) \triangleq E\{x(0, \omega)\}$, 共 分散行列 $\widehat{P}(0 \mid 0)=C_{o v}\{x(0, \omega)\}$ をもつガウス分布にし たがい， $\gamma(t, \omega)$ は，0または 1 の值をとる右連続なジ ヤンプ・マルコフ過程11)であると仮定する.このジャ ンプ・マルコフ過程が不確実な観測機構を特徴づけて おり, 以下では, interruption 過程と呼ばれている. また, $F(t), H(t)$ は $t$ に関して連続であり, 確率過程 $\gamma(t, \omega), w(t, \omega), v(t, \omega)$. および $x(0, \omega)$ は相互に独 立であると仮定する。以下では，混乱の恐れのない場 合，根元事象 $\omega$ は省略される.

一以下で，必要となるつぎの記号を定義しておく。

$$
\begin{aligned}
& P_{i j}(t, s) \triangleq P(\gamma(s)=j \mid \gamma(t)=i) \\
& q_{i j}(t) \triangleq \lim _{s \rightarrow t+0} \frac{P_{i j}(t, s)}{s-t}
\end{aligned}
$$

$\tau_{n} \triangleq$ interruption 過程 $\gamma(t)$ が $n$ 回目の

$$
\text { ジャンプをする時刻 }
$$

ここで, $s>t \geq 0, P(\cdot \mid \cdot)$ 注条件つき確率, $i, j=0,1$ $(i \neq j), n$ 注正整数である. また，定義 $(2 \cdot 4)$ の極限 $q_{i j}(t)$ の存在, その収束の $t$ に関する一様性, および $q_{i j}(t)$ の $t$ 亿関する連続性を仮定する ${ }^{11)}$. 上の定義 $(2$. 4), (2.5) 加

$$
\begin{aligned}
& \not\left(\tau_{1} \mid \gamma(0)=i\right)=q_{i j}\left(\tau_{1}\right) \exp \left\{-\int_{0}^{\tau_{1}} q_{i j}(s) d s\right\} \\
& p\left(\tau_{2 m} \mid \gamma(0)=i, \tau_{2 m-1}\right) \\
& \quad=q_{j i}\left(\tau_{2 m}\right) \exp \left\{-\int_{\tau_{2 m-1}}^{\tau_{2 m}} q_{j i}(s) d s\right\} \\
& p\left(\tau_{2 m+1} \mid \gamma(0)=i, \tau_{2 m}\right) \\
& \quad=q_{i j}\left(\tau_{2 m+1}\right) \exp \left\{-\int_{\tau_{2 m}}^{\tau_{2 m+1}} q_{i j}(s) d s\right\}
\end{aligned}
$$

ただし， $p(\cdot 1 \cdot)$ 注条件つき確率密度関数， $m$ 泟 整数, $0<\tau_{1}<\tau_{2}<\cdots, i, j,=0,1(i \neq j)$ である.

問題は，観測データ $\{y(s), 0 \leq s \leq t\}$ が与えられたと きの状態 $x(t)$ の最小分散推定值 $\hat{x}(t \mid t)$ 定見出すこと である。一般的に，最小分散推定值 $\hat{x}(t \mid t)$ 注，条件つ き期待值

$$
\hat{x}(t \mid t)=E\left\{x(t) \mid Y^{t}\right\}
$$

で与えられることが知られている。ただし， $Y^{t}$ は観測 $\{y(s), 0 \leq s<t\}$ によって生成される最小 $\sigma$-集合体で ある。

条件つき期待値の性質 ${ }^{12}$ から，

$$
\begin{aligned}
& \hat{x}(t \mid t)=E\left\{E\left\{x(t) \mid \gamma(0), \tau_{1}, \tau_{2}, \cdots, Y^{t}\right\} \mid Y^{t}\right\} \\
& \quad=\sum_{i=0}^{1}\left[E\left\{x(t) \mid \gamma(0)=i, \tau_{1} \geq t, Y^{t}\right\}\right. \\
& \quad \times P\left(\tau_{1} \geq t \mid \gamma(0)=i, Y^{t}\right) P\left(\gamma(0)=i \mid Y^{t}\right) \\
& \quad+\sum_{n=1}^{\infty} \int_{0}^{t} \int_{\tau_{1}}^{t} \ldots \int_{\tau_{n-1}}^{t} E\left\{x(t) \mid \gamma(0)=i, \tau^{n} ; \tau_{n+1} \geq t, Y^{t}\right\} \\
& \quad \times p\left(\tau^{n} \mid \gamma(0)=i, Y^{t}\right) P\left(\tau_{n+1} \geq t \mid \gamma(0)=i, \tau^{n}, Y^{t}\right) \\
& \left.\quad \times P\left(\gamma(0)=i \mid Y^{t}\right) d \tau^{n}\right]
\end{aligned}
$$

ここで, $\tau^{n} \triangleq\left(\tau_{1}, \tau_{2}, \cdots, \tau_{n}\right)$ であり, $E\{x(t) \mid r(0)=i$, $\left.\tau_{1} \geq t, Y^{t}\right\}$ および $E\left\{x(t) \mid \gamma(0)=i, \tau^{n} ; \tau_{n+1} \geq t, Y^{t}\right\}(n$ $\left.=1,2, \cdots, 0<\tau_{1}<\tau_{2}<\cdots<\tau_{n}<t\right)$ は, interruption 過程 $\{\gamma(s\}, 0 \leq s<t\}$ の值が条件として指定されているの で，通常の Kalmanフィルタを用いて得ることができ る。したがって，最小分散推定アルゴリズムの構造安 決めるには，

$$
P\left(\tau_{1} \geq t \mid \gamma(0)=i, \quad Y^{t}\right) P\left(\gamma(0)=i \mid Y^{t}\right)
$$

および

$$
\begin{aligned}
p\left(\tau^{n} \mid \gamma(0)=i, Y^{t}\right) P\left(\tau_{n+1} \geq\right. & \left.t \mid \gamma(0)=i, \tau^{n}, Y^{t}\right) \\
& \times P\left(\gamma(0)=i \mid Y^{t}\right) \\
\left(n=1,2, \cdots, 0<\tau_{1}<\tau_{2}<\right. & \left.\cdots<\tau_{n}<t\right)
\end{aligned}
$$

を生み出すアルゴリズムを導けば十分である.

\section{3. 最小分散推定アルゴリズム}

時間間隔 $[o, t)$ における interruption 過程 $\{\gamma(s)$, $0 \leq s<t\}$ のジャンプの回数を

$$
n_{\tau}{ }^{t} \triangleq \sup _{\tau_{i} \in[o, t)} i
$$

と定義し，正整数 $N$ に対して， $\Omega$ の可測部分集合

$$
B_{N}{ }^{t} \triangleq\left\{\omega \mid n_{\tau}^{t} \leq N\right\}
$$

を定義する。すなわち， $\omega \epsilon B_{N}{ }^{t}$ であれば， $\{\gamma(s, \omega)$ ， $0 \leq s<t\}$ のジャンプの回数注 $N$ 以下である.さらに， $B_{N} t$ の部分集合

$$
\begin{aligned}
& B^{t}\left(\gamma(0)=i, \tau^{n}=t^{n}\right) \triangleq\left\{\omega \mid \gamma(0, \omega)=i, \tau_{1}(\omega)\right. \\
& \left.\quad=t_{1}, \cdots, \tau_{n}(\omega)=t_{n} ; \tau_{n+1}(\omega) \geq t\right\} \\
& \quad n=0,1, \cdots \cdots, N
\end{aligned}
$$

を定義する。ただし， $\tau^{n} \triangleq\left(\tau_{1}, \cdots, \tau_{n}\right), t^{n} \triangleq\left(t_{1}, \cdots, t_{n}\right)$, $0<t_{1}<\cdots<t_{n}<t$ であり, $n=0$ では

$$
B^{t}\left(\gamma(0)=i, \tau^{0}=t^{0}\right) \triangleq\left\{\omega \mid \gamma(0, \omega)=i, \tau_{1}(\omega) \geq t\right\}
$$
とする。

ここで, $\theta \triangleq\left(\theta_{0}, \theta_{1}, \cdots, \theta_{N}\right)$ をつぎのように定義され た $N+1$ 次元の確率变数とする。すなおち，

$$
\theta(\omega)=\left(i, t_{1}, t_{2}, \cdots, t_{n}, \tau^{*}, \tau^{*}, \cdots, \tau^{*}\right) \text {, }
$$




$$
\begin{gathered}
\left(\omega \epsilon B^{t}\left(\gamma(0)=i, \tau^{n}=t^{n}\right) \text { のとき }\right) \\
n=0,1, \cdots, N
\end{gathered}
$$

$\theta(\omega)=(0,0, \cdots 0) \quad\left(\omega \epsilon \Omega-B_{N}{ }^{t}\right.$ のとき $)$

ただし， $\tau^{*}$ は任意の固定された負の定数, $0<t_{1}<\cdots$ $<t_{n}<t$ であり, $n=0$ では,

$\left.\left(i, t_{1}, t_{2}, \cdots, t_{n}, \tau^{*} \tau^{*}, \cdots, \tau^{*}\right)\right|_{n=0} \triangleq\left(i, \tau^{*}, \tau^{*}, \cdots, \tau^{*}\right)$ とする. 以上の定義から，もし $\theta \neq(0,0, \cdots, 0)$ なる $\theta$ を 1 つ与えたなら゙, $\{r(s), 0 \leq s<t\}$ は完全に規定され ることになる。したがって, 根元事象 $\omega$ を $\Omega$ の部分集 合 $B_{N}{ }^{t}$ に制限して考えるならば，時間間隔 $[0, t)$ にお いて, $\theta(\omega)$ を式 (2・1), (2·2) で表現される線形系の $N$ +1 次元未知パラメータ・ベクトルとみなすことがで きる.よって，Lainiotis の公式により，

$$
p\left(\theta \mid B_{N}^{t}, Y^{t}\right)=\frac{L(t, \theta) p\left(\theta \mid B_{N}^{t}\right)}{\int_{R^{N+1}} L(t, \theta) p\left(\theta \mid B_{N}^{t}\right) d \theta}
$$

が成り立つ.ただし，

$$
\begin{aligned}
& L(t, \theta) \triangleq \exp \left\{\int_{0}^{t} \gamma(s, \theta) \hat{x}^{\prime}(s \mid s, \theta) H^{\prime}(s) R^{-1}(s) d y(s)\right. \\
& \left.\quad-\frac{1}{2} \int_{0}^{t} \gamma(s, \theta)\|H(s) \hat{x}(s \mid s, \theta)\|{ }^{2} R^{-1}(s) d s\right\}(3 \cdot 5) \\
& \hat{x}(s \mid s, \theta) \triangleq E\left\{x(s) \mid \theta, Y^{s}\right\}
\end{aligned}
$$

であり, $\gamma(s, \theta(\omega))$ は $\theta(\omega)$ に対応する $\gamma(s, \omega)$ として 定義され， $R^{N+1}$ は $N+1$ 次元ユークリッド空間であ る. $(3 \cdot 3)$ の定義から， $B_{N}{ }^{t}$ が与えられたときの $\theta$ の 条件つき確率密度関数 $p\left(\theta \mid B_{N}{ }^{t}\right)$ はつぎのように表わ される。

$$
\begin{aligned}
& p\left(\theta \mid B_{N}^{t}\right)=\sum_{i=0}^{1} P\left(\gamma(0)=i \mid B_{N} t\right) \delta\left(\theta_{0}-i\right)\left\{P\left(\tau_{1} \geq t \mid \gamma(0)=i, B_{N}^{t}\right) \delta\left(\theta_{1}-\tau^{*}\right) \cdots \delta\left(\theta_{N}-\tau^{*}\right)\right. \\
& \quad+\sum_{n=1}^{N-1} p\left(\theta^{n} \mid \theta_{0}=i, B_{N}^{t}\right) P\left(\tau_{n+1} \geq t \mid \gamma(0)=i, \tau_{n}=\theta_{n}, B_{N}^{t}\right) \delta\left(\theta_{n+1}-\tau^{*}\right) \cdots \delta\left(\theta_{N}-\tau^{*}\right) \\
& \left.\quad+p\left(\theta^{N} \mid \theta_{0}=i, B_{N}^{t}\right)\right\}
\end{aligned}
$$

ただし， $\delta(\cdot)$ はディラックのデルタ関数， $\theta^{n} \triangleq\left(\theta_{1}, \cdots, \theta_{n}\right)$ であり, $0<\theta_{1}<\cdots<\theta_{n}<t$ に対して,

$$
p\left(\theta^{n} \mid \theta_{0}=i, B_{N}{ }^{t}\right)=p\left(\tau^{n} \mid \gamma(0)=i, B_{N}{ }^{t}\right) \mid \begin{gathered}
\tau_{1}=\theta_{1} \\
\vdots \\
\tau_{n}=\theta_{n}
\end{gathered} .
$$

その他の $\theta^{n}$ に対しては,

$$
p\left(\theta^{n} \mid \theta_{0}=i, B_{N}{ }^{t}\right)=0
$$

である、また，事後確率密度関数 $p\left(\theta \mid B_{N}{ }^{t}, Y^{t}\right)$ もつぎのように表わされる。

$$
\begin{aligned}
& p\left(\theta \mid B_{N}^{t}\right)=\sum_{i=0}^{1} P\left(\gamma(0)=i \mid B_{N}^{t}, Y^{t}\right) \delta\left(\theta_{0}-i\right)\left\{P\left(\tau_{1} \geq t \mid \gamma(0)=i, B_{N}{ }^{t}, Y^{t}\right) \delta\left(\theta_{1}-\tau^{*}\right) \cdots \delta\left(\theta_{N}-\tau^{*}\right)\right. \\
& \quad+\sum_{n=1}^{N-1} p\left(\theta^{n} \mid \theta_{0}=i, B_{N}^{t}, Y^{t}\right) P\left(\tau_{n+1} \geq t \mid \gamma(0)=i, \tau^{n}=\theta^{n}, B_{N^{t}}, Y^{t}\right) \delta\left(\theta_{n+1}-\tau^{*}\right) \cdots \delta\left(\theta_{N}-\tau^{*}\right) \\
& \left.\quad+p\left(\theta^{N} \mid \theta_{0}=i, B_{N}{ }^{t}, Y^{t}\right)\right\}
\end{aligned}
$$

ただし， $0<\theta_{1}<\cdots<\theta_{n}<t$ に対して，

$$
p\left(\theta^{n} \mid \theta_{0}=i, B_{N}{ }^{t}, Y^{t}\right)=p\left(\tau^{n} \mid \gamma(0)=i, B_{N}{ }^{t}, Y^{t}\right) \mid \begin{gathered}
\tau_{1}=\theta_{1} \\
\vdots \\
\tau_{n}=\theta_{n}
\end{gathered}
$$

であり，その他の $\theta^{n}$ に対しては，

$$
p\left(\theta^{n} \mid \theta_{0}=i, B_{N}{ }^{t}, Y^{t}\right)=0
$$

である.式 (3.6)，(3.8) を式 (3.4) に代入し，その両辺を比較することによって，

$$
\begin{aligned}
& P\left(\tau_{1} \geq t \mid \gamma(0)=i, B_{N}{ }^{t}, Y^{t}\right) P\left(\gamma(0)=i \mid B_{N}{ }^{t}, Y^{t}\right) \\
& \quad=\frac{\left.L(t, \theta)\right|_{\theta=\left(i, \tau^{*}, \cdots, \tau^{*}\right)} P\left(\tau_{1} \geq t \mid \gamma(0)=i, B_{N} t\right) P\left(\gamma(0)=i \mid B_{N} t\right)}{L_{N}(t)}
\end{aligned}
$$

および，

$$
\begin{aligned}
& p\left(\theta^{n} \mid \theta_{0}=i, B_{N}{ }^{t}, Y^{t}\right) P\left(\tau_{n+1} \geq t \mid \gamma(0)=i, \tau^{n}=\theta^{n}, B_{N}{ }^{t}, Y^{t}\right) P\left(\gamma(0)=i \mid B_{N}{ }^{t}, Y^{t}\right) \\
& \quad=\frac{\left.L(t, \theta)\right|_{\theta=\theta^{*}} p\left(\theta^{n} \mid \theta_{0}=i, B_{N} t\right) P\left(\tau_{n+1} \geq t \mid \gamma(0)=i, \tau_{n}=\theta_{n}, B_{N} t\right) P\left(\gamma(0)=i \mid B_{N}{ }^{t}\right)}{L_{N}(t)}
\end{aligned}
$$

を得る。ただし， $\theta^{*} \triangleq\left(i, \theta_{1}, \cdots, \theta_{n}, \tau^{*}, \cdots, \tau^{*}\right), 0<\theta_{1}<\cdots<\theta_{n}<t, n=1,2, \cdots, N, i=0,1$,

$$
L_{N}(t) \triangleq \int_{R^{N+1}} L(t, \theta) p\left(\theta \mid B_{N}^{t}\right) d \theta \text {. }
$$


定義 $(3 \cdot 2),(3 \cdot 3)$ から, 以下では, $B^{t}\left(\gamma(0), \tau^{n}\right)$ の 上において, $\theta_{0}, \theta_{i}(i=1,2, \cdots, n)$ の代わりに, $\gamma(0)$, $\tau_{i}(i=1,2, \cdots, n)$ を用いることにする. したがって, 次 式を定義しておく.

$$
\begin{aligned}
& L\left(t, B^{t}\left(\gamma(0)=i, \tau^{n}\right)\right) \\
& \left.\quad \triangleq L(t, \theta)\right|_{\left.\theta_{=\left(i, \tau_{1}\right.}, \cdots, \tau_{n}, \tau^{*}, \cdots, \tau^{*}\right)}
\end{aligned}
$$

ただし， $n=0,1, \cdots, N, i=0,1$. 式 $(3 \cdot 6),(3 \cdot 7)$ および $(3 \cdot 13)$ によって, 式 $(3 \cdot 12)$ は

$$
\begin{aligned}
& L_{N}(t)=\sum_{i=0}^{1}\left\{L\left(t, B^{t}\left(\gamma(0)=i, \tau^{0}\right)\right) P\left(\gamma(0)=i \mid B_{N}{ }^{t}\right) P\left(\tau_{1} \geq t \mid \gamma(0)=i, B_{N} t\right)\right. \\
& \quad+\sum_{n=1}^{N} \int_{0}^{t} \int_{\tau_{1}}^{t} \cdots \int_{\tau_{n-1}}^{t} L\left(t, B^{t}\left(\gamma(0)=i, \tau^{n}\right)\right) p\left(\tau^{n} \mid \gamma(0)=i, B_{N} t\right) P\left(\tau_{n+1} \geq t \mid \gamma(0)=i, \tau_{n}, B_{N} t\right) \\
& \left.\quad \times P\left(\gamma(0)=i \mid B_{N} t\right) d \tau^{n}\right\}
\end{aligned}
$$

となる. 式 $(3 \cdot 5),(3 \cdot 10) \sim(3 \cdot 14)$ によって，つぎの補題 1 が成り立つ.

\section{《補題 1》}

$$
\begin{aligned}
& 『 P\left(\tau_{1} \geq t \mid \gamma(0)=i, B_{N}{ }^{t}, Y^{t}\right) P\left(\gamma(0)=i \mid B_{N}{ }^{t}, Y^{t}\right) \\
& =\frac{L\left(t, B^{t}\left(\gamma(0)=i, \tau^{0}\right)\right) P\left(\tau_{1} \geq t \mid \gamma(0)=i, B_{N}{ }^{t}\right) P\left(\gamma(0)=i \mid B_{N}{ }^{t}\right)}{L_{N}(t)} \\
& p\left(\tau^{n} \mid \gamma(0)=i, B_{N}^{t}, Y^{t}\right) P\left(\tau_{n+1} \geq t \mid \gamma(0)=i, \tau^{n}, B_{N}{ }^{t}, Y^{t}\right) P\left(\gamma(0)=i \mid B_{N}{ }^{t}, Y^{t}\right) \\
& \quad=\frac{L\left(t, B^{t}\left(\gamma(0)=i, \tau^{n}\right)\right) p\left(\tau^{n} \mid \gamma(0)=i, B_{N}{ }^{t}\right) P\left(\tau_{n+1} \geq t \mid \gamma(0)=i, \tau_{n}, B_{N}\right) P\left(\gamma(0)=i \mid B_{N}{ }^{t}\right)}{L_{N}(t)}
\end{aligned}
$$

ただし $, 0<\tau_{1}<\cdots<\tau_{n}<t, n=1,2, \cdots, N, i=0,1$,

$$
\begin{aligned}
L\left(t, B^{t}\left(\gamma(0)=i, \tau^{0}\right)\right) & =\exp \left\{\int_{0}^{t} i \cdot \hat{x}_{i}{ }^{\prime}\left(s \mid s, \tau^{0}\right) H^{\prime}(s) R^{-1}(s) d y(s)-\frac{1}{2} \int_{0}^{t} i \cdot \| H(s) \hat{x}_{i}\left(s \mid s, \tau^{0}\right) \mid{ }^{2} R^{-1}(s) d s\right\} \\
L\left(t, B^{t}\left(\gamma(0)=i, \tau^{n}\right)\right) & =\exp \left\{\int_{0}^{t} \gamma_{i}\left(s, \tau^{n}\right) \hat{x}_{i}{ }^{\prime}\left(s \mid s, \tau^{n}\right) H^{\prime}(s) R^{-1}(s) d y(s)\right. \\
& \left.-\frac{1}{2} \int_{0}^{t} \gamma_{i}\left(s, \tau^{n}\right)\left\|H(s) \hat{x}_{i}{ }^{\prime}\left(s \mid s, \tau^{n}\right)\right\|^{2} R^{-1}(s) d s\right\} .
\end{aligned}
$$

であり， $L_{N}(t)$ は式 $(3 \cdot 14)$ で与えられる.ここで, $\gamma_{i}\left(s, \tau^{n}\right)$ は $\gamma(0)=i, 0<\tau_{1}<\cdots<\tau_{n}<t \leq \tau_{n+1}$ である ときの $\gamma(s)(0 \leq s<t)$ を示しており,

$$
\begin{aligned}
& \hat{x}_{i}\left(s \mid s, \tau^{0}\right) \triangleq E\left\{x(s) \mid Y^{s}, \gamma(0)=i, \tau_{1} \geq t\right\} \quad(3 \cdot 19) \\
& \hat{x}_{i}\left(s \mid s, \tau^{n}\right) \triangleq E\left\{x(s) \mid Y^{s}, \gamma(0)=i, \tau^{n} ; \tau_{n+1} \geq t\right\}
\end{aligned}
$$
である・』
条件つき確率の性質から，

$$
\begin{gathered}
P\left(\tau_{1} \geq t \mid \gamma(0)=i, B_{N}{ }^{t}\right) P\left(\gamma(0)=i \mid B_{N} t\right) \\
=\frac{P\left(\tau_{1} \geq t \mid \gamma(0)=i\right) P(\gamma(0)=i)}{P\left(B_{N} t\right)}
\end{gathered}
$$

が成り立つ. 同様にして, $n=1,2, \cdots, N$ に対して，

$$
\begin{gathered}
p\left(\tau^{n} \mid \gamma(0)=i, B_{N}^{t}\right) P\left(\tau_{n+1} \geq t \mid \gamma(0)=i, \tau_{n}, B_{N} t\right) P\left(\gamma(0)=i \mid B_{N} t\right) \\
=\frac{\left.p\left(\tau^{n} \mid \gamma(0)=i\right)\right) P\left(\tau_{n+1} \geq t \mid \gamma(0)=i, \tau_{n}\right) P(\gamma(0)=i)}{P\left(B_{N} t\right)}
\end{gathered}
$$

が成り立つ. 式(3.20)，(3·21) をそれぞれ式（3·15)，(3·16) に代入すれば，つぎの補題 2 を得る.

\section{《補題 2》.}

$$
\begin{aligned}
& 『 P\left(\tau_{1} \geq t \mid \gamma(0)=i, B_{N}{ }^{t}, Y^{t}\right) P\left(\gamma(0)=i \mid B_{N^{t}}, Y^{t}\right)=\frac{L\left(t, B^{t}\left(\gamma(0)=i, \tau^{0}\right)\right) P\left(\tau_{1} \geq t \mid \gamma(0)=i\right) P(\gamma(0)=i)}{L_{N}{ }^{\prime}(t)} \\
& p\left(\tau^{n} \mid \gamma(0)=i, B_{N}{ }^{t}, Y^{t}\right) P\left(\tau_{n+1} \geq t \mid \gamma(0)=i, \tau^{n}, B_{N} t, Y^{t}\right) P\left(\gamma(0)=i \mid B_{N} t, Y^{t}\right) \\
& =\frac{L\left(t, B^{t}\left(\gamma(0)=i, \tau^{n}\right)\right) p\left(\tau^{n} \mid \gamma(0)=i\right) P\left(\tau_{n+1} \geq t \mid \gamma(0)=i, \tau_{n}\right) P(\gamma(0)=i)}{L_{N}^{\prime}(t)}
\end{aligned}
$$

ただし $, 0<\tau_{1}<\cdots<\tau_{n}<t, n=1,2, \cdots, N, i=0,1$,

$$
\begin{aligned}
& L_{N}^{\prime}(t) \triangleq \sum_{i=0}^{1}\left\{L\left(t, B^{t}\left(\gamma(0)=i, \tau^{0}\right)\right) P\left(\tau_{1} \geq t \mid \gamma(0)=i\right) P(\gamma(0)=i)+\sum_{n=1}^{N} \int_{0}^{t} \int_{\tau_{1}}^{t} \ldots \int_{\tau_{n-1}}^{t} L\left(t, B^{t}\left(\gamma(0)=i, \tau^{n}\right)\right)\right. \\
& \left.\times p\left(\tau^{n} \mid \gamma(0)=i\right) P\left(\tau_{n+1} \geq t \mid \gamma(0)=i, \tau_{n}\right) P(\gamma(0)=i) d \tau^{n}\right\} \rrbracket
\end{aligned}
$$


すべての有限な $t(>0)$ に対して，

$\lim _{N \rightarrow \infty} B_{N}{ }^{t}=\Omega-\Lambda^{t}, \quad P\left(\Lambda^{t}\right)=0$

が成り立つ11ので，

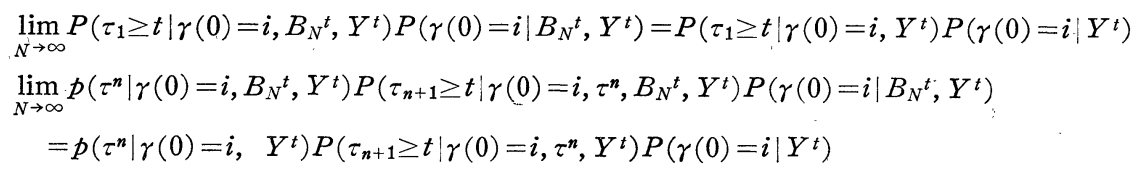

が, 確率 1 で成り立つ. さらに, 式 (3.24) からわか るように, $L_{N}{ }^{\prime}(t)$ は正で, $N$ に関して単調増大であ り，式(3·22)，(3·23）の分子はともに $N$ に無関係であ ることから, 式 $(3 \cdot 22)$, (3.23) の両辺性 $N \rightarrow \infty$ のと き収束する.したがって, 式 $(3.22) \sim(3 \cdot 27)$ により,

$$
\begin{aligned}
& P\left(\tau_{1} \geq t \mid \gamma(0)=i, Y^{t}\right) P\left(\gamma(0)=i \mid Y^{t}\right) \\
& \quad=\frac{L\left(t, B^{t}\left(\gamma(0)=i, \tau^{0}\right)\right) P\left(\tau_{1} \geq t \mid \gamma(0)=i\right) P(\gamma(0)=i)}{L(t)}
\end{aligned}
$$

および

$$
\begin{aligned}
& p\left(\tau^{n} \mid \gamma(0)=i, Y^{t}\right) P\left(\tau_{n+1} \geq t \mid \gamma(0)=i, \tau^{n}, Y^{t}\right) P\left(\gamma(0)=i \mid Y^{t}\right) \\
& =\frac{L\left(t, B^{t}\left(\gamma(0)=i, \tau^{n}\right)\right) p\left(\tau^{n} \mid \gamma(0)=i\right) P\left(\tau_{n+1} \geq t \mid \gamma(0)=i, \tau_{n}\right) P(\gamma(0)=i)}{L(t)}
\end{aligned}
$$

が，確率1で成り立つことがわかる．ただし， $n=1,2, \cdots$,

$$
\begin{aligned}
& L(t) \triangleq \sum_{i=0}^{1}\left\{L\left(t, B^{t}\left(\gamma(0)=i, \tau^{0}\right)\right) P\left(\tau_{1} \geq t \mid \gamma(0)=i\right) P(\gamma(0)=i)+\sum_{n=1}^{\infty} \int_{0}^{t} \int_{\tau_{1}}^{t} \ldots \int_{\tau_{n-1}}^{t} L\left(t, B^{t}\left(\gamma(0)=i, \tau^{n}\right)\right)\right. \\
& \left.\quad \times p\left(\tau^{n} \mid \gamma(0)=i\right) P\left(\tau_{n+1} \geq t \mid \gamma(0)=i, \tau_{n}\right) P(\gamma(0)=i) d \tau^{n}\right\} \\
& \text { ここで, 式 }(2 \cdot 6) \text { より, } \\
& P\left(\tau_{1} \geq t \mid \gamma(0)=i\right)=\exp \left\{-\int_{0}^{t} q_{i j}(s) d s\right\} \\
& P\left(\tau_{n+1} \geq t \mid \gamma(0)=i, \tau_{n}\right)=\exp \left\{-\int_{\tau_{n}}^{t} q_{i j}(s) d s\right\} \quad(n=2 m \text { のとき }) \\
& =\exp \left\{-\int_{\tau_{n}}^{t} q_{j i}(s) d s\right\} \quad(n=2 m-1 \text { のとき })
\end{aligned}
$$

$$
\begin{aligned}
p\left(\tau^{n} \mid \gamma(0)=i\right)=q_{i j}\left(\tau_{1}\right) \exp \left\{-\int_{0}^{\tau_{1}} q_{i j}(s) d s\right\} q_{j i}\left(\tau_{2}\right) \exp \left\{-\int_{\tau_{1}}^{\tau_{2}} q_{j i}(s) d s\right\} \cdots q_{j i}\left(\tau_{n}\right) \exp \left\{-\int_{\tau_{n-1}}^{\tau_{n}} q_{j i}(s) d s\right\} \\
\quad(n=2 m \text { のとき) } \\
=q_{i j}\left(\tau_{1}\right) \exp \left\{-\int_{0}^{\tau_{1}} q_{i j}(s) d s\right\} q_{i i}\left(\tau_{2}\right) \exp \left\{-\int_{\tau_{1}}^{\tau} q_{j i}(s) d s\right\} \cdots q_{i j}\left(\tau_{n}\right) \exp \left\{-\int_{\tau_{n-1}}^{\tau_{n}} q_{i j}(s) d s\right\} \\
\quad(n=2 m-1 \text { のとき) }
\end{aligned}
$$

ただし $, i, j=0,1(i \neq j), m=1,2$,

式 $(2 \cdot 8),(3 \cdot 28) \sim(3 \cdot 33)$ によて，最小分散推定 アルゴリズムの構造注完全に定まった．以上の結果を まとめると，つぎの定理を得る。

【定理】『最小分散推定 $\hat{x}(t \mid t)$ は，次式で与えられ る。

$$
\begin{aligned}
& \hat{x}(t \mid t)=\sum_{i=0}^{1}\left\{\hat{x}_{i}\left(t \mid t, \tau^{0}\right) P\left(\tau_{1} \geq t \mid \gamma(0)=i, Y^{t}\right)\right. \\
& \times P\left(\gamma(0)=i \mid Y^{t}\right)+\sum_{n=1}^{\infty} \int_{0}^{t} \int_{\tau_{1}}^{t} \cdots \int_{\tau_{n-1}}^{t} \hat{x}_{i}\left(t \mid t, \tau^{n}\right)
\end{aligned}
$$

$$
\begin{aligned}
& \times p\left(\tau^{n} \mid \gamma(0)=i, Y^{t}\right) P\left(\tau_{n+1} \geq t \mid \gamma(0)=i, \tau^{n}, Y^{t}\right) \\
& \left.\times P\left(\gamma(0)=i \mid Y^{t}\right) d \tau^{n}\right\}
\end{aligned}
$$

ここで， $\hat{x}_{i}\left(t \mid t, \tau^{n}\right)(i=0,1, n=0,1,2, \cdots)$ は式 (3. 19）で定義されたもので，通常の Kalmanフィルタに より与えられ, $P\left(\tau_{1} \geq t \mid \gamma(0)=i, Y^{t}\right) P\left(\gamma(0)=i \mid Y^{t}\right)$, $p\left(\tau^{n} \mid \gamma(0)=i, Y^{t}\right) P\left(\tau_{n+1} \geq t \mid \gamma(0)=i, \tau^{n}, Y^{t}\right) \cdot P(\gamma(0)$ $\left.=i \mid Y^{t}\right)$ は，それぞれ式 $(3 \cdot 28)$ ，(3・29)で与えられる. また, 式 $(3 \cdot 28),(3 \cdot 29)$ における $L\left(t, B^{t}(\gamma(0)=i\right.$, $\left.\left.\tau^{0}\right)\right), L\left(t, B^{t}\left(\gamma(0)=i, \tau^{n}\right)\right)$ は，それだれ式(3.17), (3. 
18）で与えられる・』

最小分散推定值を与える式 (3·34) の右辺は無限個 の総和となっているので, 式 (3.34) によって最小分 散推定 $\hat{x}(t \mid t)$ を求めることは，ある正整数 $N$ に対し て $P\left(B_{N}{ }^{t}\right)=1$ となる場合, すなわち interruption 過 程 $\{\gamma(s), 0 \leq s<t\}$ が確率 1 で $N$ 回以下のジャンプし かしない場合を除いて，実行不可能である。したがっ て，実行可能な近似最小分散推定アルゴリズム它次節 において提案する。

\section{4. 近似推定アルゴリズム}

本節では, 以下の 2 つ特別な場合について, 近似 最小分散推定アルゴリズムを提案する。

\section{（1）終端時刻が有限で固定されている場合}

終端時刻を $T$ とする. そのとき，十分小さい正数 $\varepsilon$ に対して， $N_{0}$ を $N \geq N_{0}$ ならば，つぎの不等式

$$
P\left(B_{N}^{T}\right) \geq 1-\varepsilon
$$

が成り立つような正整数として定義する。なお, $\lim _{N \rightarrow \infty} P$ $\left(B_{N}{ }^{T}\right)=1$ が成り立つ11)ので, そのような正整数 $N_{0}$ は 常に存在する.上の定義から, interruption 過程 $\gamma(t)$ は時間間隔 $[0, T)$ において, 確率 $\varepsilon$ 以下で， $N_{0}$ 回以 上のジャンプをすることがわかる。したがって，

$$
x^{*}(t \mid t) \triangleq E\left\{x(t) \mid Y^{t}, B_{N_{0}}{ }^{t}\right\}
$$

を $x(t)$ の推定值として採用すれば， $1-\varepsilon$ 以上の確率 で， $x(t)$ の最適推定值を得ることができる。式 $(4 \cdot 2)$ の推定值は

$$
\begin{gathered}
x^{*}(t \mid t)=E\left\{E\left\{x(t) \mid r(0), \tau^{N_{0}}, Y^{t}, B_{N_{0}}{ }^{t}\right\} \mid Y^{t}, B_{N_{0}}{ }^{t}\right\} \\
=\sum_{i=0}^{1}\left\{\hat{x}_{i}\left(t \mid t, \tau^{0}\right) P\left(\tau_{1} \geq t \mid \gamma(0)=i, B_{N_{0}}{ }^{t}, Y^{t}\right)\right. \\
\times P\left(\gamma(0)=i \mid B_{N_{0}}{ }^{t}, Y^{t}\right) \\
+\sum_{n=1}^{N_{0}} \int_{0}^{t} \int_{\tau_{1}}^{t} \cdots \int_{\tau_{n-1}}^{t} \hat{x}_{i}\left(t \mid t, \tau^{n}\right) p\left(\tau^{n} \mid \gamma(0)=i,\right. \\
\left.\quad B_{N_{0}}{ }^{t}, Y^{t}\right) P\left(\tau_{n+1} \geq t \mid \gamma(0)=i, \tau^{n}, B_{N_{0}}{ }^{t}, Y^{t}\right) \\
\left.\times P\left(\gamma(0)=i \mid B_{N_{0}}{ }^{t}, Y^{t}\right) d \tau^{n}\right\}
\end{gathered}
$$

により, 式(3・19) および補題 $2\left(N=N_{0}\right)$ を用いて計 算される。

\section{（2）終端時刻が無限大の場合}

以下の議論の簡単化のため, interruption 過程 $\gamma(t)$ は定常であると仮定する. ある正整数 $N_{0}$ と十分小さ い正数 $\varepsilon$ に対して， $t_{0}$ を $t \leq t_{0}$ ならば，つぎの不等式

$$
\min _{i=0,1} P\left(B_{N_{0}}{ }^{t} \mid \gamma(0)=i\right) \geq 1-\varepsilon
$$

が成り立つような正数として定義する。ここで, $N_{0}$ と には，それぞれ計算機容量および推定值のよさに関係 する.以下では, 終端時刻が無限大の場合に対する近
似最小分散推定アルゴリズムを得るために，つぎの仮 定をする。

[仮定］『各時刻 $t\left(>t_{0}\right)$ に対して, 条件つき確率密 度関数 $p\left(x\left(t-t_{0}\right) \mid Y^{t-t_{0}}\right)$ 注

$$
\begin{aligned}
& \text { 平均値 }=x^{*}\left(t-t_{0} \mid t-t_{0}\right) \triangleq E\left\{x\left(t-t_{0}\right) \mid Y^{t-t_{0}}\right\} \\
& \text { 共分散 }=P^{*}\left(t-t_{0} \mid t-t_{0}\right) \triangleq C o_{v}\left\{x\left(t-t_{0}\right) \mid Y^{t-t_{0}}\right\}
\end{aligned}
$$

をもつガウス分布にしたがう・』

この仮定のもとに, 近似最小分散推定 $x^{*}(t \mid t)$ は, つぎの手順で得られる。

(i) 式(4.4)の条件から， $0 \leq t \leq t_{0}$ では本節の (1)で 提案したアルゴリズムを適用し， $x^{*}(t \mid t), P *(t \mid t)$, $p(1 \mid t) \triangleq P\left(\gamma(t)=1 \mid B_{N_{0}}{ }^{t}, Y^{t}\right)$ を求める.ただし， $P^{*}(t \mid t), p(1 \mid t)$ は大れぞれ式(4-3) と同様にして， 容易に求められる。

(ii) $t_{0}<t$ 、では, interruption 過程の定常性および $(4 \cdot 4),(4 \cdot 5)$ により $, x^{*}\left(t-t_{0} \mid t-t_{0}\right), P^{*}\left(t-t_{0} \mid t-\right.$ $\left.t_{0}\right), P\left(1 \mid t-t_{0}\right)$ を初期值とし，( $\left.\mathrm{i}\right)$ のアルゴリズム 学用いて， $x^{*}(t \mid t), P^{*}(t \mid t), p(1 \mid t)$ 等求める。

\section{5. 結論}

観測がランダムに中断される場合の線形連続時間系 に対する最小分散推定，およびその近似推定アルゴリ ズムが，示された，最適推定值を得るために，無限個 の総和を必要とするので，最適推定アルゴリズム（3・ 34）は一般的に性実行不可能である。しかし，inter ruption 過程が，ある正整数 $N$ に対して, 確率 1 で, $N$ 回以下のジャンプしかとない場合には， 4 節の前半で 提案した近似推定アルゴリズムが最適なものとなり， 実行可能となる。なお，近似推定フルゴリズムを用い る際に，ジャンプ・マルコフ過程の特性によって決ま るある水準にまで，ジャンプの生ずる時刻を量子化し なけ机ばいけないことを注意しておく。

また，本論文での議論とほとえど同様にして，ジャ ンプ・マルコフ過程が任意の有限個の值をとる場合や， ジャンプ・マルコフ過程が観測方程式だけでなく，シ ステム方程式にも含まれる場合の最小分散推定アルゴ リズムを導くことができることを注意しておく。

最後に，近似推定アルゴリズムの導出において，わ れわれの用いた仮定 $(4 \cdot 5)$ ，の妥当性に関する理論的裏 づけは事実上不可能であると思われるが，われわれの 行なった計算機シミュレーションでは，近似推定值が ほとえど最適推定值と一致しており，実用的観点から は，仮定 $(4.5)$ が容認されるのではないかと考えられ る。 


\section{参 考文・献}

1) R. E. Kalman: A new approach to linear filtering and prediction problems, Trans. ASME, J. Basic Engrg., ser. D, Vol. 82, 35/45, (1960)

2) R.E. Kalman and R.S. Bucy: New results in linear filtering and prediction theory, Trans. ASME, J. Basic Engrg., ser. D, Vol. 83, 95/108, (1961)

3) N.E. Nahi: Optimal recursive estimation with uncertain observation, IEEE Trans. Inform. Theory, Vol. IT-15, 457/462, (1969)

4) M.Z. Dajani and A.P. Sage: Nonlinear sequential algorithms for estimation under uncertainty, Information Sciences, Vol. 2, No. 4, 379/393, (1970)

5) A. G. Jaffer and S. C. Gupta: Recursive Bayesian estimation with uncertain observation, IEEE Trans. Inform. Theory, Vol. IT-17, 614/616, (1971)

6) A.G. Jaffer and S.C. Gupta: Optimal sequential estimation of discrete processes with Markov interrupted observations, IEEE Trans. Autom. Contr., Vol. AC-16, 471/475, (1971)

7) Y. Sawaragi, T. Katayama and. S. Fujishige: Sequential state estimation with interrupted observation, Information and Control, Vol. 21, No. 1, 56/71, (1972)

8）椹木，片山，藤重：不確実な観測機構を有する線形系の 状態推定, 計測自動制御学会論文集, 第 8 巻, 第 4 号, 499/507, (1972)

9) D.G. Lainiotis: Optimal adaptive estimation: Structure and parameter adaptation, IEEE Trans. Autom. Contr., Vol. AC-16, 160/170, (1971)

10) D. G. Lainiotis: Joint detection, estimation and system identification, Information and Control, Vol. 19, No. 1, 75/92, (1971)

11) I. I. Gikhman and A.V. Skorokhod: Introduction to the Theory of Random Processes. Philadelphia; W. B. Saunders, (1969)

12) J. L. Doob: Stochastic Processes. New York; John Wiley, (1953) 